\title{
Optimisation Model for Proportioning the Aggregates of High Strength Laterised Concrete
}

\author{
Dr. Udeme Hanson Iron* and Professor Dahunsi, Bamidele I.O.** \\ *Lecturer, Department of Civil Engineering, Faculty of Engineering, University of Uyo, Nigeria \\ ***Professor, Department of Civil Engineering, Faculty of Technology, University of Ibadan, Nigeria \\ DOI: 10.29322/IJSRP.11.06.2021.p11457 \\ http://dx.doi.org/10.29322/IJSRP.11.06.2021.p11457
}

\begin{abstract}
Laterite, a typically red or reddish brown soil found in abundance in the tropics has been used to partially or wholly replace sand in concrete. Resulting laterised concretes are generally known to exhibit low strengths. In this study, the production of high strength laterised concretes using superplasticiser was experimented. Scheffe's simplex theory based on $(6,2)$ simplex lattice was used to optimize the mix proportions for the crushing strength of high strength laterised concrete produced using Conplast SP 430 superplasticiser (a sulphonated naphthalene formaldehyde admixture). Mathematical models were developed for the mix proportioning of high strength laterised concrete. All strengths predicted by the developed mathematical model are in good agreement with their corresponding experimentally observed values. Using the model, the mix proportions for any targeted strength of hardened concrete is easily evaluated with the help of the computer programme. A computer programme written in Q-BASIC language is also provided for speedy generation of the mix proportions for the targeted strength.
\end{abstract}

Index Terms- Crushing strength, Laterized concrete, Optimisation, Simplex lattice, Superplasticiser.

\section{INTRODUCTION}

L aterised concrete has found immense use of recent for the construction of low-cost buildings. The use of this material is known to reduce the cost of structures due to the abundance of laterite within the tropics and sub tropics (Udoeyo et al, 2006). Studies on the usage of this material have shown very encouraging results (Orangun 1981). Although used, its usage has hitherto been limited to structures of lower strengths. Moreover, in most previous studies on laterised concretes, the traditional mix design methods with its cumbersome nature were utilized. The need to produce laterised concretes of higher strengths and also eliminate the errors and cumbersome nature of the traditional mix design methods informed the will to embark on this study in order to develop an optimization model for accurate and effective proportioning of high strength laterised concretes. The strength of concrete is a function of the proportions of the ingredients that make up the concrete. The task of accurate proportioning still remains a problem to concreters. Eliminating this problem is the focus of this study.

The crushing strength $\left(\mathrm{f}_{\mathrm{c}}\right)$, which is the most desired and convenient to measure quality of hardened concrete, is optimized in this study. It is evaluated using:

$\mathrm{f}_{\mathrm{c}}=\mathrm{P} / \mathrm{A}$

Where

$\mathrm{P}$ is the maximum crushing load at failure and

A is the cross sectional area of the specimen (cube).

\section{MAteRIALS AND MethodS}

\subsection{Materials}

Materials used in this study were water, cement, sand, laterite, coarse granite aggregate, and Conplast SP 430 superplasticizer.

Laterite: The laterite was obtained from burrow pits in Uyo, Akwa Ibom State of Nigeria. Its specific gravity was 2.62 and it was a zone 3 aggregate according to BS 882:1992 classification. Its elemental contents were analysed at the Ministry of Science and Technology laboratory in Akwa Ibom State and presented in Table 1. Its fineness modulus and coefficient of uniformity were 2.33 and 2.40 respectively.

Sand: The sand was obtained from Ikpa River in Uyo and prepared according to BS 882 - 103:1992 requirements. It had a specific gravity of 2.65, fineness modulus of 2.37 and coefficient of uniformity of 2.83. It was a zone 2 aggregate with reference to BS $882-$ 103:1992 grading system. The particle size distribution of the sand and laterite are shown in figure 1.

Coarse aggregate: The coarse aggregate was coarse granite stones obtained from Akamkpa in Cross River State, Nigeria. It had a specific gravity of 2.71 , impact value of $13.15 \%$ and crushing value of 21.43 with a size ranging between $10 \mathrm{~mm}$ and $20 \mathrm{~mm}$. 
Cement: Cement was "UNICEM" Brand of Portland Limestone cement conforming to type 1 cement. It is manufactured in Cross River State of Nigeria.

Superplasticizer: The high performance Conplast SP 430 superplasticizing admixture belonging to the sulphonated naphthalene formaldehyde ( $\mathrm{SNF}$ ) class was used for this work. It had a specific gravity of 1.18 with alkali content typically less than $55 \mathrm{~g}$. $\mathrm{Na}_{2} \mathrm{O}$ equivalent/litre of the admixture.

Water: Water used throughout the work was obtained from the Civil Engineering laboratory in the University of Uyo, Nigeria. It was clean and conformed to BS 3148 requirements.

\section{$2.2 \quad$ Methods}

The base mix ratios used for the generation of the simplex design points were as a result of preliminary research findings about laterised concretes. Batching of the concrete constituents (water, cement, sand, laterite, coarse aggregate and superplasticizer) was by weight while mixing of the constituents was manually done. The $150 \mathrm{~mm}$ concrete cubes were prepared to conform to the requirements of BS 1881: Part 108:1983 and BS 1881: Part 111:1983. Each cube was stored in a damp environment for twenty-four hours after which it was demoulded and stored in a curing tank at a temperature of $20 \pm 2^{\circ} \mathrm{C}$ for 28 days. The $28^{\text {th }}$ day crushing strengths of the cubes were determined using a digital compression testing machine to the requirements of BS 1881: Part 115: 1986 and Part 116:1983. The digital machine recorded both the maximum load at failure in kiloNewtons $(\mathrm{kN})$ and the crushing strength produced in $\mathrm{N} / \mathrm{mm}^{2}$. These were recorded and the strengths were however confirmed using the formula in eqn. 1

\section{THE OPTIMIZATION THEORY}

An optimization theory developed by Scheffe (1958) was used in the optimization of the concrete strengths. The strength of concrete primarily is dependent on the proper proportioning of its constituents and adequate proportioning is the crux of all mixes designs.

\subsection{The Simplex}

Simplex is the structural representation (shape) of the line or planes joining the assumed positions of the constituent materials (atoms) making the mixture (Jackson 1983). Scheffe (1958) considered mixtures of which their studied property depended on the proportions of the constituents. A typical example is the crushing strength of concrete and its relationship with the mix proportions of water/cement ratio, cement, fine aggregate and coarse aggregate.

Suppose a mixture has a total of $q$ components and $X_{i}$ is the proportion of the component of $i^{\text {th }}$ ingredient in the mixture such that $X_{i} \geq 0(i=1-6)$

Assuming the mixture to be a unit quantity, then the sum of all the proportions of the components must be unity.

Considering this experiment:

$\mathrm{x}_{1}+\mathrm{x}_{2}+\mathrm{x}_{3}+\mathrm{x}_{4}+\mathrm{x}_{5}+\mathrm{x}_{6}=1$

Or

$\sum \mathrm{x}_{\mathrm{i}}=1$

Where

$\mathrm{x}_{1}=$ is the proportion of water/cement ratio

$\mathrm{x}_{2}=$ is the proportion of cement

$\mathrm{x}_{3}=$ is the proportion of sand

$\mathrm{x}_{4}=$ is the proportion of laterite

$\mathrm{x}_{5}=$ is the proportion of coarse aggregate

$\mathrm{X}_{6}=$ is the proportion of superplasticizer

Hence the factor space is a regular $(q-1)$ dimensional simplex

\subsection{Simplex lattices:}

In a $(\mathrm{q}-1)$ - dimensional simplex, for binary system, $(q=2)$ the simplex is a straight-line segment having only two points of connectivity and for $q=3$, the regular 2-simplex is an equilateral triangle with its interior. Each point in the triangle corresponds to a certain composition of the mixture system, and conversely each composition is represented by one distinct point. For a four-component $(q=4)$ mixture, the regular simplex is a tetrahedron where each vertex represents a binary system. Beyond 4 components, the regular simplex becomes a regular polygon with corresponding vertices.

Scheffe's simplex-lattice designs provide a uniform scatter of points over the $(q-1)$ simplex. The points form a $(q, n)$ lattice on the simplex where $q$ is the number of mixture components; $n$ is the degree of polynomial. Simplex-lattice designs are saturated and for each component there exist $(n+1)$ similar levels from 0 to 1 ; i.e. $\mathrm{x}_{\mathrm{i}}=0,1 / n, 2 / n,---, 1$ and all possible combinations are derived with such values of component concentrations. Scheffe (1958) also showed that the property studied (e.g. crushing strength of concrete) is assumed to be a continuous function of certain arguments and with a sufficient accuracy it can be approximated by a polynomial. Moreover, he proved that a polynomial of degree $n$ in $q$ variables has $C_{q+n}^{n}$ points on the lattice but by using the relationship in eqn. (3), the number of points can be reduced to $C^{n}{ }_{q+n-1}$.

This implies that the number of points

For a $(6,2)$ lattice, which this experiment is based the number of points equals: 


$$
\begin{aligned}
C_{q+n-1}^{n} & =\frac{q(q+1)--(q+n-1)}{n !} \\
& =\frac{6(6+1)}{2 * 1}=21
\end{aligned}
$$

\subsection{Experiment design points:}

The assumption in eqn. 3 makes it impossible to use the normal mix ratios such as 1:2:4, 1:1.5:3 or 1:3:6 at a given water/cement ratio and binder/superplasticizer ratio. This necessitates the transformation of the actual components (ingredients) proportions to meet the criterion. Such transformed ratios $\mathrm{x}_{\mathrm{i}}(i=1-6)$ for each experimental point are called "pseudo components". For actual components $Z$, the pseudo component $\mathrm{X}$ is given by:

$X=A Z$

Where

$A$ is the inverse of $Z$ matrix. Similarly the inverse transformation from pseudo components to actual components is expressed as:

$Z=B X$

Where

$B$ is the inverse of $A$ matrix. The pseudo components $X$ and the actual components $Z$ utilized for the design points are presented in table 2 .

\subsection{Preparation of Crushing Strength Test Cubes}

Cube specimens of size $150 \mathrm{~mm} \times 150 \mathrm{~mm} \times 150 \mathrm{~mm}$ were prepared for testing. Each cube was prepared to conform to the requirements of BS 1881: part 108: 1983 and BS1881: Part111: 1983. The inside of each cube mould was lightly oiled before use. The mould was then filled in three approximately equal layers of concrete and each layer was compacted manually using a $25 \mathrm{~mm}$ square steel punner and 35 strokes of the rod was rendered on each layer. On complete compaction, the top of the concrete was trowelled smooth and given an identification mark. The cube was then stored in a damp environment for twenty-four hours before demoulding. After 24hours the cube was demoulded carefully to avoid damage to the arises. The demoulded cubes were submerged and stored in a curing tank at a temperature of $20 \pm 2^{\circ} \mathrm{C}$ for 28 days before testing. After twenty-eight days curing, the cubes were removed from the curing tank and cleaned of water. They were weighed before testing.

\section{i. Table 1: Elemental analysis of the laterite samples}

\begin{tabular}{llll}
\hline Element & Sample A\% & Sample B\% & Sample C\% \\
\hline Aluminium & 3.61 & 2.92 & 3.05 \\
Silicon & 49.46 & 47.44 & 49.39 \\
Iron & 28.59 & 30.61 & 27.72 \\
Manganese & 6.12 & 9.38 & 10.32 \\
Lead & 1.25 & 1.21 & 1.34 \\
Zinc & 9.78 & 7.40 & 7.01 \\
Nickel & 0.85 & 0.89 & 1.03 \\
Copper & 0.34 & 0.15 & 0.14 \\
\hline
\end{tabular}

\begin{tabular}{|c|c|c|c|c|c|c|c|c|c|c|c|c|c|}
\hline \multirow[t]{2}{*}{ No. } & \multicolumn{6}{|c|}{ Pseudo Components } & \multirow{2}{*}{$\begin{array}{l}\text { Responses } \\
\text { yobs }^{\text {ons }}\end{array}$} & \multicolumn{6}{|c|}{ Actual Components } \\
\hline & $\mathrm{X}_{1}$ & $\mathrm{X}_{2}$ & $\mathrm{X}_{3}$ & $\mathrm{X}_{4}$ & $\mathrm{X}_{5}$ & $\mathrm{X}_{6}$ & & $\mathrm{Z}_{1}$ & $\mathrm{Z}_{2}$ & $\mathrm{Z}_{3}$ & $\mathrm{Z}_{4}$ & $\mathrm{Z}_{5}$ & $\mathrm{Z}_{6}$ \\
\hline 1. & 1 & 0 & 0 & 0 & 0 & 0 & $\mathrm{Y}_{1}$ & 0.36 & 1 & 0.5 & 0.5 & 2.5 & 0.035 \\
\hline 2. & 0 & 1 & 0 & 0 & 0 & 0 & $\mathrm{Y}_{2}$ & 0.38 & 1 & 1 & 0.5 & 3 & 0.03 \\
\hline 3. & 0 & 0 & 1 & 0 & 0 & 0 & $\mathrm{Y}_{3}$ & 0.4 & 1 & 1.2 & 0.8 & 4 & 0.025 \\
\hline 4. & 0 & 0 & 0 & 1 & 0 & 0 & $Y_{4}$ & 0.42 & 1 & 0.5 & 1 & 3.5 & 0.02 \\
\hline 5. & 0 & 0 & 0 & 0 & 1 & 0 & $\mathrm{Y}_{5}$ & 0.45 & 1 & 0.5 & 1 & 3 & 0.015 \\
\hline 6. & 0 & 0 & 0 & 0 & 0 & 1 & $Y_{6}$ & 0.35 & 1 & 1.2 & 0.6 & 3.6 & 0.04 \\
\hline 7. & 0.5 & 0.5 & 0 & 0 & 0 & 0 & $\mathrm{Y}_{12}$ & 0.37 & 1 & 0.75 & 0.5 & 2.75 & 0.0325 \\
\hline 8. & 0.5 & 0 & 0.5 & 0 & 0 & 0 & $\mathrm{Y}_{13}$ & 0.38 & 1 & 0.85 & 0.65 & 3.25 & 0.03 \\
\hline 9. & 0.5 & 0 & 0 & 0.5 & 0 & 0 & $\mathrm{Y}_{14}$ & 0.39 & 1 & 0.5 & 0.75 & 3 & 0.0275 \\
\hline 10. & 0.5 & 0 & 0 & 0 & 0.5 & 0 & $Y_{15}$ & 0.405 & 1 & 0.5 & 0.75 & 2.75 & 0.025 \\
\hline 11. & 0.5 & 0 & 0 & 0 & 0 & 0.5 & $Y_{16}$ & 0.355 & 1 & 0.85 & 0.55 & 3.05 & 0.0375 \\
\hline 12. & 0 & 0.5 & 0.5 & 0 & 0 & 0 & $Y_{23}$ & 0.39 & 1 & 1.1 & 0.65 & 3.5 & 0.0275 \\
\hline 13. & 0 & 0.5 & 0 & 0.5 & 0 & 0 & $\mathrm{Y}_{24}$ & 0.4 & 1 & 0.75 & 0.75 & 3.25 & 0.025 \\
\hline
\end{tabular}

Table 2: Pseudo and Actual components for points 1-30 (6,2) mixture 


\begin{tabular}{|c|c|c|c|c|c|c|c|c|c|c|c|c|c|c|c|c|}
\hline 14. & 0 & 0.5 & 0 & 0 & 0.5 & 0 & $\mathrm{Y}_{25}$ & 0.415 & 1 & & 0.75 & 0.75 & & 3 & & 0.0225 \\
\hline 15. & 0 & 0.5 & 0 & 0 & 0 & 0.5 & $\mathrm{Y}_{26}$ & 0.365 & 1 & & 1.1 & 0.55 & & 3.3 & & 0.035 \\
\hline 16. & 0 & 0 & 0.5 & 0.5 & 0 & 0 & $\mathrm{Y}_{34}$ & 0.41 & 1 & & 0.85 & 0.9 & & 3.75 & & 0.0225 \\
\hline 17. & 0 & 0 & 0.5 & 0 & 0.5 & 0 & $\mathrm{Y}_{35}$ & 0.425 & 1 & & 0.85 & 0.9 & & 3.5 & & 0.02 \\
\hline 18. & 0 & 0 & 0.5 & 0 & 0 & 0.5 & $\mathrm{Y}_{36}$ & 0.375 & 1 & & 1.2 & 0.7 & & ii. & 3.8 & 0.0325 \\
\hline 19. & 0 & 0 & 0 & 0.5 & 0.5 & 0 & $\mathrm{Y}_{45}$ & 0.435 & 1 & & 0.5 & 1 & & 3.25 & & 0.0175 \\
\hline 20. & 0 & 0 & 0 & 0.5 & 0 & 0.5 & $Y_{46}$ & 0.385 & 1 & & 0.85 & 0.8 & & 3.55 & & 0.03 \\
\hline 21. & 0 & 0 & 0 & 0 & 0.5 & 0.5 & $Y_{56}$ & 0.4 & iii. & 1 & 0.85 & 0.8 & & 3.3 & & 0.0275 \\
\hline \multicolumn{17}{|c|}{ Control Points } \\
\hline 22. & 0.2 & 0.2 & 0.2 & 0.1 & 0.2 & 0.1 & $\mathrm{C}_{11}$ & 0.395 & 1 & & 0.81 & 0.72 & & 3.21 & & 0.027 \\
\hline 23. & 0 & 0.3 & 0.2 & 0.3 & 0 & 0.2 & $\mathrm{C}_{22}$ & 0.39 & 1 & & 0.93 & 0.73 & & 3.47 & & 0.028 \\
\hline 24. & 0.1 & 0.1 & 0.1 & 0.4 & 0.2 & 0.1 & $\mathrm{C}_{33}$ & 0.407 & 1 & & 0.69 & 0.84 & & 3.31 & & 0.024 \\
\hline 25. & 0.3 & 0.3 & 0.3 & 0 & 0.1 & 0 & $\mathrm{C}_{44}$ & 0.387 & 1 & & 0.86 & 0.64 & & 3.15 & & 0.0285 \\
\hline 26. & 0 & 0 & 0 & 0.3 & 0.3 & 0.4 & $\mathrm{C}_{55}$ & 0.401 & 1 & & 0.78 & 0.84 & & 3.39 & & 0.0265 \\
\hline 27. & 0.2 & 0.4 & 0 & 0 & 0.2 & 0.2 & $\mathrm{C}_{66}$ & 0.384 & 1 & & 0.84 & 0.62 & & 3.02 & & 0.03 \\
\hline 28. & 0.2 & 0 & 0.2 & 0.3 & 0.1 & 0.2 & $\mathrm{C}_{77}$ & 0.393 & iv. & 1 & 0.78 & 0.78 & & 3.37 & & 0.0275 \\
\hline 29. & 0.1 & 0.1 & 0.5 & 0.1 & 0.1 & 0.1 & $\mathrm{C}_{88}$ & 0.396 & 1 & & 0.97 & 0.76 & & 3.56 & & 0.0265 \\
\hline 30. & 0.2 & 0.2 & 0.2 & 0.2 & 0 & 0.2 & $\mathrm{C}_{99}$ & 0.382 & 1 & & 0.88 & v. & 0.64 & 3.32 & & 0.03 \\
\hline
\end{tabular}

Legend: $\mathrm{y}_{i}, \mathrm{y}_{i j}(i=1-6$ and $j=2-6)$ are the response, that is the expected compressive strength of the concrete.

\subsection{The Simplex canonical Polynomials}

Scheffe (1958) described the mixture properties by reduced polynomials obtained subject to the condition in equation [3]. The properties studied in the reduced polynomial are real-valued functions on the simplex. They are referred to, as "RESPONSES". In this study, the target strength of the concrete would be the response.

Scheffe (1958) also showed that a polynomial function of degree $n$ in $q$ variables $x_{1}, x_{2}, x_{3, \ldots} \ldots x_{q}$ subject to equation [3] will be called a $(q, n)$ polynomial. Accordingly if the response $(\hat{y})$ is a function of the components (or variables) $x_{1}, x_{2}, x_{3}, x_{4}, \ldots x_{q}$, then the polynomial is of the form;

$\hat{y}=b_{0}+\sum_{1 \leq i \leq q} b_{i} X_{i}+\sum_{1 \leq i \leq j \leq q} b_{i j} X_{i} X_{j}+\sum_{1 \leq i \leq j \leq k \leq q} b_{i j k} X_{i} X_{j} X_{k}+\sum b i_{1} i_{2}---i_{n} X i_{1} X i_{2} X i_{n}$

Where all $b_{\mathrm{s}}$ are constant coefficients. He also showed that the number of coefficients in eqn [6] is given by $\mathrm{C}_{\mathrm{q}+\mathrm{n}}^{\mathrm{n}}$ corresponding to the number of points on the lattice. These coefficients can be reduced to

$\mathrm{C}_{\mathrm{q}+\mathrm{n}-1}$ when the condition in eqn. [3] is applied. For example if from eqn. [3], we let.

$$
X_{q}=1-\sum_{i=1}^{q} X_{i}
$$

Then substituting the value of $\mathrm{x}_{q}$ into eqn [6], the number of coefficient $b_{i}$ will reduce to

$$
C^{n}{ }_{q+n-1} \text { or } \frac{q(q+1) \ldots \ldots . .(q+n-1)}{n !} \text {. }
$$

This implies that the number of the coefficients equals to a $(q, n)$ lattice. Another implication is that the values of a $(q, n)$ polynomial can be assigned arbitrarily on a $(q, n)$ lattice and its values on the simplex uniquely determined.

In order to have a manageable number of coefficients, Scheffe (1958) avoided high-degree polynomials. He also showed that the general low-degree polynomial of degree $n$ and $q$ variables subject to eqn. [3] may be written as:

$\hat{y}=\alpha_{1} X_{1}+\alpha_{2} X_{2}+\alpha_{3} X_{3}+\alpha_{4} X_{4}+\alpha_{5} X_{5}+\alpha_{6} X_{6}+\alpha_{12} X_{1} X_{2}+\alpha_{13} X_{1} X_{3}+\alpha_{14} X_{1} X_{4}$

$+\alpha_{15} X_{1} X_{5}+\alpha_{16} X_{1} X_{6}+\alpha_{23} X_{2} X_{3}+\alpha_{24} X_{2} X_{4}+\alpha_{25} X_{2} X_{5}+\alpha_{26} X_{2} X_{6}+\alpha_{34} X_{3} X_{4}$

$+\alpha_{35} X_{3} X_{5}+\alpha_{36} X_{3} X_{6}+\alpha_{45} X_{4} X_{5}+\alpha_{46} X_{4} X_{6}+\alpha_{56} X_{5} X_{6}$

The coefficients $\alpha_{\mathrm{i}}$ and $\alpha_{\mathrm{ij}}$ are shown by Scheffe (1958) to be

$\alpha_{i}=y_{i}$

And

$\alpha_{i j}=4 y_{i j}-2 y_{i}-2 y_{j}$ 


\section{RESULTS AND ANALYSIS}

The results of the crushing strength test based on Scheffe's $(6,2)$ simplex lattice experimental design are presented in Table 3. The results covered twenty one experimental points and nine control points.

\subsection{The Regression Equation}

Using eqns. [10], [11] and Table 2, the coefficients of the $(6,2)$ polynomial of eqn. [9] are determined as follows:

$\alpha_{1}=\mathrm{y}_{1}=48.7, \alpha_{2}=\mathrm{y}_{2}=48.9, \alpha_{3}=\mathrm{y}_{3}=42.4, \alpha_{4}=\mathrm{y}_{4}=40.3, \alpha_{5}=\mathrm{y}_{5}=37.7$ and

$\alpha_{6}=\mathrm{y}_{6}=44.1$.

$\alpha_{12}=4(50.1)-2(48.7)-2(48.9)=5.20$

$\alpha_{13}=4(47.3)-2(48.7)-2(42.4)=7.00$

$\alpha_{14}=4(44.3)-2(48.7)-2(40.3)=-0.80$

$\alpha_{15}=4(48.0)-2(48.7)-2(37.7)=19.20$

$\alpha_{16}=4(47.1)=2(48.7)-2(44.1)=2.80$

$\alpha_{23}=4(42.3)-2(48.9)-2(42.4)=-13.40$

$\alpha_{24}=4(42.6)-2(48.9)-2(40.3)=-8.00$

$\alpha_{25}=4(39.1)-2(48.9)-2(37.7)=-16.80$

$\alpha_{26}=4(43.7)=2(48.9)-2(44.1)=-11.20$

$\alpha_{34}=4(40.1)-2(42.4)-2(40.3)=-5.00$

$\alpha_{35}=4(39.3)-2(42.4)-2(37.7)=-3.00$

$\alpha_{36}=4(44.0)=2(42.4)-2(44.1)=3.00$

$\alpha_{45}=4(34.2)-2(40.3)-2(37.7)=-19.20$

$\alpha_{46}=4(41.2)=2(40.3)-2(44.1)=-4.00$

$\alpha_{56}=4(42.7)=2(37.7)-2(44.1)=7.20$

Thus from equation (9)

$y c=\quad 48.7 x_{1}+48.9 x_{2}+42.4 x_{3}+40.3 x_{4}+37.7 x_{5}+44.1 x_{6}+5.2 x_{1} x_{2}+7.0 x_{1} x_{3}-0.8 x_{1} x_{4}+19.2 x_{1} x_{5}+2.8 x_{1} x_{6}-13.4 x_{2} x_{3}-8.0 x_{2} x_{4}-$ $16.8 x_{2} x_{5}-11.2 x_{2} x_{6}-5.0 x_{3} x_{4}-3.0 x_{3} x_{5}+3.0 x_{3} x_{6}-19.2 x_{4} x_{5}-4.0 x_{4} x_{6}+7.2 x_{5} x_{6} \ldots \ldots \ldots .$.

Eqn.[12] is the Scheffes' mathematical model for the optimization of the crushing strength of high strength laterised concrete based on $(6,2)$ lattice experimentation.

\subsection{Test of the Adequacy of the model equation:}

The student $-\mathrm{t}$ test was used to test the adequacy of the model equation developed. The experimental results of the nine control points were subjected to the student $-\mathrm{t}$ test. The $\mathrm{t}-$ table $(\mathrm{t}=2.97)$ was far greater than all the $\mathrm{t}-\mathrm{calculated}$ for all the nine control points as shown in table 4 ; hence the model equation was proved adequate.

To finally arrive at the optimization, a computer programme was written in $\mathrm{Q}-$ Basic for interpretation. By running the programme, the model predicted a maximum crushing strength of $50.05 \mathrm{~N} / \mathrm{mm} 2$ corresponding to an optimum mix proportion of $1: 2: 5: 9$ with a water/cement ratio of 0.66 .

For any desired strength, the mix proportions can easily be obtained by running the Q - Basic programme with the target strength as input. If there is no matching proportion, the computer quickly notifies the user.

Table 3: Analysis of Crushing Strength of High Strength Laterized Concrete

\begin{tabular}{|c|c|c|c|c|c|c|c|c|}
\hline Exp. No. & $\begin{array}{l}\text { Max. Load } \\
(\mathrm{kN})\end{array}$ & $\begin{array}{l}\text { Response } \\
\mathrm{y}_{\mathrm{r}} \\
\left(\mathrm{N} / \mathrm{mm}^{2}\right)\end{array}$ & $\mathrm{y}_{\mathrm{r}}^{2}$ & $\Sigma \mathrm{y}_{\mathrm{r}}$ & $\begin{array}{l}\bar{y} \\
\left(\mathrm{~N} / \mathrm{mm}^{2}\right)\end{array}$ & $\begin{array}{l}\text { Response } \\
\text { symbol }\end{array}$ & $\Sigma y_{r}^{2}$ & $\mathrm{~S}_{\mathrm{i}}^{2}$ \\
\hline $1 \mathrm{~A}$ & 1080.8 & 48.0 & 2307.41 & 97.3 & 48.7 & $\mathrm{Y}_{1}$ & 4736.81 & 0.7854 \\
\hline $1 \mathrm{~B}$ & 1109.0 & 49.3 & 2429.39 & & & & & \\
\hline $2 \mathrm{~A}$ & 1081.9 & 48.1 & 2312.11 & 97.8 & 48.9 & $Y_{2}$ & 4788.17 & 1.4037 \\
\hline $2 B$ & 1119.6 & 49.8 & 2476.06 & & & & & \\
\hline $3 \mathrm{~A}$ & 934.9 & 41.6 & 1726.49 & 84.9 & 42.4 & $Y_{3}$ & 3603.12 & 1.5645 \\
\hline $3 B$ & 974.7 & 43.3 & 1876.62 & & & & & \\
\hline $4 \mathrm{~A}$ & 896.8 & 39.9 & 1588.64 & 80.7 & 40.3 & $\mathrm{Y}_{4}$ & 3255.82 & 0.4737 \\
\hline $4 B$ & 918.7 & 40.8 & 1667.18 & & & & & \\
\hline $5 \mathrm{~A}$ & 849.5 & 37.8 & 1425.48 & 75.4 & 37.7 & $\mathrm{Y}_{5}$ & 2843.26 & 0.0052 \\
\hline $5 B$ & 847.2 & 37.7 & 1417.77 & & & & & \\
\hline $6 \mathrm{~A}$ & 986.1 & 43.8 & 1920.78 & 88.2 & 44.1 & $Y_{6}$ & 3893.72 & 0.1747 \\
\hline $6 \mathrm{~B}$ & 999.4 & 44.4 & 1972.94 & & & & & \\
\hline
\end{tabular}




\begin{tabular}{|c|c|c|c|c|c|c|c|c|}
\hline $7 \mathrm{~A}$ & 1129.6 & 50.2 & 2520.49 & 100.2 & 50.1 & $\mathrm{Y}_{12}$ & 5022.26 & 0.0174 \\
\hline $7 \mathrm{~B}$ & 1125.4 & 50.0 & 2501.78 & & & & & \\
\hline $8 \mathrm{~A}$ & 1086.0 & 48.3 & 2329.67 & 94.6 & 47.3 & $Y_{13}$ & 4472.74 & 1.9470 \\
\hline $8 \mathrm{~B}$ & 1041.6 & 46.3 & 2143.07 & & & & & \\
\hline 9A & 1016.7 & 45.2 & 2041.83 & 88.6 & 44.3 & $\mathrm{Y}_{14}$ & 3922.31 & 1.6602 \\
\hline 9B & 975.7 & 43.4 & 1880.48 & & & & & \\
\hline $10 \mathrm{~A}$ & 1077.3 & 47.9 & 2292.49 & 95.9 & 48.0 & $\mathrm{Y}_{15}$ & 4600.34 & 0.0128 \\
\hline 10B & 1080.9 & 48.0 & 2307.84 & & & & & \\
\hline $11 \mathrm{~A}$ & 1036.8 & 46.1 & 2123.37 & 94.2 & 47.1 & $Y_{16}$ & 4439.76 & 2.0990 \\
\hline $11 \mathrm{~B}$ & 1082.9 & 48.1 & 2316.39 & & & & & \\
\hline $12 \mathrm{~A}$ & 945.9 & 42.0 & 1767.36 & 84.5 & 42.3 & $\mathrm{y}_{23}$ & 3572.67 & 0.1008 \\
\hline $12 \mathrm{~B}$ & 956.0 & 42.5 & 1805.31 & & & & & \\
\hline $13 \mathrm{~A}$ & 954.5 & 42.4 & 1799.64 & 85.2 & 42.6 & у24 & 3629.20 & 0.0616 \\
\hline 13B & 962.4 & 42.8 & 1829.56 & & & & & \\
\hline $14 \mathrm{~A}$ & 855.1 & 38.0 & 1444.34 & 78.1 & 39.1 & y25 & 3055.02 & 2.2661 \\
\hline 14B & 903.0 & 40.1 & 1610.68 & & & & & \\
\hline $15 \mathrm{~A}$ & 992.0 & 44.1 & 1943.83 & 87.5 & 43.7 & $\mathrm{y}_{26}$ & 3825.85 & 0.2497 \\
\hline 15B & 976.1 & 43.4 & 1882.02 & & & & & \\
\hline $16 \mathrm{~A}$ & 917.4 & 40.8 & 1662.46 & 80.2 & 40.1 & y34 & 3219.03 & 0.8712 \\
\hline 16B & 887.7 & 39.5 & 1556.57 & & & & & \\
\hline $17 \mathrm{~A}$ & 872.0 & 38.8 & 1501.99 & 78.6 & 39.3 & $\mathrm{y} 35$ & 3086.39 & 0.5501 \\
\hline 17B & 895.6 & 39.8 & 1584.39 & & & & & \\
\hline $18 \mathrm{~A}$ & 973.2 & 43.3 & 1870.85 & 88.0 & 44.0 & $\mathrm{y} 36$ & 3870.33 & 1.0690 \\
\hline $18 \mathrm{~B}$ & 1006.1 & 44.7 & 1999.48 & & & & & \\
\hline $19 \mathrm{~A}$ & 778.5 & 34.6 & 1197.16 & 68.4 & 34.2 & y45 & 2339.30 & 0.3236 \\
\hline 19B & 760.4 & 33.8 & 1142.14 & & & & & \\
\hline $20 \mathrm{~A}$ & 939.2 & 41.7 & 1742.41 & 82.4 & 41.2 & y46 & 3398.00 & 0.5548 \\
\hline $20 \mathrm{~B}$ & 915.5 & 40.7 & 1655.59 & & & & & \\
\hline $21 \mathrm{~A}$ & 956.2 & 42.5 & 1806.06 & 85.4 & 42.7 & $\mathrm{y}_{56}$ & 3642.85 & 0.0648 \\
\hline 21B & 964.3 & 42.9 & 1836.79 & & & & & \\
\hline $22 \mathrm{~A}$ & 955.9 & 42.5 & 1804.93 & 85.9 & 43.0 & $\mathrm{C}_{11}$ & 3690.80 & 0.4439 \\
\hline $22 \mathrm{~B}$ & 977.1 & 43.4 & 1885.88 & & & & & \\
\hline $23 \mathrm{~A}$ & 942.3 & 41.9 & 1753.93 & 82.7 & 41.3 & $\mathrm{C}_{22}$ & 3418.57 & 0.5832 \\
\hline $23 \mathrm{~B}$ & 918.0 & 40.8 & 1664.64 & & & & & \\
\hline $24 \mathrm{~A}$ & 935.7 & 41.6 & 1729.45 & 81.8 & 40.9 & $\mathrm{C}_{33}$ & 3344.78 & 0.9738 \\
\hline 24B & 904.3 & 40.2 & 1615.33 & & & & & \\
\hline $25 \mathrm{~A}$ & 1004.7 & 44.7 & 1993.92 & 89.9 & 44.9 & $\mathrm{C}_{44}$ & 4038.17 & 0.1568 \\
\hline $25 \mathrm{~B}$ & 1017.3 & 45.2 & 2044.25 & & & & & \\
\hline $26 \mathrm{~A}$ & 901.1 & 40.0 & 1603.91 & 81.1 & 40.5 & $\mathrm{C}_{55}$ & 3286.01 & 0.4651 \\
\hline $26 \mathrm{~B}$ & 922.8 & 41.0 & 1682.09 & & & & & \\
\hline $27 \mathrm{~A}$ & 1017.3 & 45.2 & 2044.25 & 88.4 & 44.2 & $\mathrm{C}_{66}$ & 3906.65 & 2.1172 \\
\hline 27B & 971.0 & 43.2 & 1862.40 & & & & & \\
\hline $28 \mathrm{~A}$ & 955.0 & 42.4 & 1801.53 & 86.5 & 43.3 & $\mathrm{C}_{77}$ & 3746.14 & 1.3668 \\
\hline $28 \mathrm{~B}$ & 992.2 & 44.1 & 1944.61 & & & & & \\
\hline $29 \mathrm{~A}$ & 960.6 & 42.7 & 1822.72 & 85.5 & 42.8 & $\mathrm{C}_{88}$ & 3658.37 & 0.0114 \\
\hline $29 \mathrm{~B}$ & 964.0 & 42.8 & 1835.65 & & & & & \\
\hline $30 \mathrm{~A}$ & 957.2 & 42.5 & 1809.84 & 87.3 & 43.6 & $\mathrm{C}_{99}$ & 3812.90 & 2.4494 \\
\hline $30 \mathrm{~B}$ & 1007.0 & 44.8 & 2003.06 & & \multirow{2}{*}{\multicolumn{4}{|c|}{24.8229}} \\
\hline$\Sigma$ & & & & & & & & \\
\hline
\end{tabular}

Table 4: $t$ - statistics for the nine control points

\begin{tabular}{llllll}
\hline S/No. & $\begin{array}{l}\text { Control } \\
\text { Point }\end{array}$ & Yexperiment & $\mathbf{Y}_{\text {theoretical }}$ & $\Delta \mathbf{Y}$ & $\mathbf{t}$ \\
\hline $\mathbf{1 .}$ & $\mathrm{C} 11$ & 43.0 & 43.2 & - & - \\
& & & & 0.2 & 0.48 \\
\hline
\end{tabular}




\begin{tabular}{|c|c|c|c|c|c|}
\hline 2. & $\mathrm{C} 22$ & 41.3 & 41.4 & $\begin{array}{l} \\
0.1\end{array}$ & $\begin{array}{l}- \\
0.26\end{array}$ \\
\hline 3. & C33 & 39.9 & 40.9 & 1.0 & 2.30 \\
\hline 4. & C44 & 44.9 & 45.6 & $\overline{0}-\overline{0}$ & $-\overline{1.93}$ \\
\hline 5. & C55 & 40.5 & 39.7 & 0.8 & 2.23 \\
\hline 6. & C66 & 44.2 & 45.0 & $\overline{0} .8$ & $-\overline{2.05}$ \\
\hline 7. & C77 & 43.3 & 42.7 & 0.6 & 1.47 \\
\hline 8. & C88 & 42.8 & 42.3 & 0.5 & 1.11 \\
\hline 9. & C99 & 43.6 & 43.9 & $\overline{0}-\overline{3}$ & $-\overline{0.75}$ \\
\hline
\end{tabular}

\subsection{Programme Testing and Test Results:}

Programme B.1: A-Q-Basic programme that optimizes the crushing strength proportions of High Strength Laterized Concrete

100 REM A Q - BASIC PROGRAMME THAT OPTIMISES SPLC MIX PROPORTIONS

110 REM VARIABLES USED ARE

120 REM X1, X2, X3, X4, X5, X6, Z1, Z2, Z3, Z4, Z5, Z6, ymax, yout, yin

130 REM MODEL USED: SPLC CRUSHING STRENGTH MODEL, EQN (4.19)

140 REM

150 REM MAIN PROGRAMME BEGINS

160 LET COUNT $=1$

170 CLS

180 GOSUB 210

190 END

200 REM END OF MAIN PROGRAMME

210 REM PROCEDURES BEGINS

220 LET $y \max =0$

230 PRINT

240 REM

250 PRINT "A COMPUTER MODEL FOR COMPUTING SPLC MIX PROPORTIONS"

260 PRINT "CORRESPONDING TO A REQUIRED CRUSHING STRENGTH"

270 REM

280 REM

290 PRINT

300 INPUT "ENTER DESIRED STRENGTH"; yin

310 GOSUB 570

320 FOR X1 $=0$ TO 1 STEP .01

330 FOR X2 $=0$ TO $1-$ X1 STEP .01

340 FOR X3 = 0 TO $1-$ X1 - X2 STEP .01

350 FOR X4 = 0 TO $1-$ X1 - X2 - X3 STEP .01

360 FOR X5 = 0 TO $1-\mathrm{X} 1-\mathrm{X} 2-\mathrm{X} 3-\mathrm{X} 4$ STEP .01

370 LET X6 = 1 - X1 - X2 - X3 - X4 - X5

380 LET yout $=47.7 * \mathrm{X} 1+47.5 * \mathrm{X} 2+41.5 * \mathrm{X} 3+40.8 * \mathrm{X} 4+36.6 * \mathrm{X} 5+44.1 * \mathrm{X} 6+9.8 * \mathrm{X} 1 * \mathrm{X} 2+8.9 * \mathrm{X} 1 * \mathrm{X} 3+.5 * \mathrm{X} 1 *$ $\mathrm{X} 4+16.7 * \mathrm{X} 1 * \mathrm{X} 5+6 * \mathrm{X} 1 * \mathrm{X} 6-9.3 * \mathrm{X} 2 * \mathrm{X} 3-3.8 * \mathrm{X} 2 * \mathrm{X} 4-9.8 * \mathrm{X} 2 * \mathrm{X} 5-7.9 * \mathrm{X} 2 * \mathrm{X} 6-5.5 * \mathrm{X} 3 * \mathrm{X} 4-0 * \mathrm{X} 3 * \mathrm{X} 5+$ $5.7 * \mathrm{X} 3 * \mathrm{X} 6-17.6 * \mathrm{X} 4 * \mathrm{X} 5-5.5 * \mathrm{X} 4 * \mathrm{X} 6+10.4 * \mathrm{X} 5 * \mathrm{X} 6$

390 GOSUB 620

$400 \mathrm{IF}(\mathrm{ABS}$ (yin - yout) <=.001) THEN 410 ELSE 430

410 LET COUNT $=$ COUNT +1

420 GOSUB 650

430 NEXT X5

440 NEXT X4

450 NEXT X3

460 NEXT X2

470 NEXT X1

480 PRINT

$490 \mathrm{IF}$ (COUNT > 0) THEN GOTO 500 ELSE GOTO 540

500 PRINT "THE MAXIMUM CRUSHING STRENGTH PREDICTABLE" 
510 PRINT "BY THIS MODEL IS"; ymax; "N/SQ.MM."

520 SLEEP (2)

530 GOTO 560

540 PRINT "SORRY! DESIRED STRENGTH OUT OF RANGE OF MODEL"

550 SLEEP 2

560 RETURN

570 REM PROCEDURE PRINT HEADING

580 REM

590 PRINT "COUNT X1 X2 X3 X4 X5 X6 Y Z1 Z2 Z3 Z4 Z5 Z6"

600 REM

610 RETURN

620 REM PROCEDURE CHECK MAX

$630 \mathrm{IF}$ ymax < yout THEN ymax = yout ELSE ymax = ymax

640 RETURN

650 REM PROCEDURE OUT RESULTS

660 LET Z1 $=.36 * \mathrm{X} 1+.38 * \mathrm{X} 2+.4 * \mathrm{X} 3+.42 * \mathrm{X} 4+.45 * \mathrm{X} 5+.35 * \mathrm{X} 6$

670 LET Z2 $=\mathrm{X} 1+\mathrm{X} 2+\mathrm{X} 3+\mathrm{X} 4+\mathrm{X} 5+\mathrm{X} 6$

680 LET Z3 $=.5 * \mathrm{X} 1+1 * \mathrm{X} 2+1.2 * \mathrm{X} 3+.5 * \mathrm{X} 4+.5 * \mathrm{X} 5+1.2 * \mathrm{X} 6$

690 LET Z4 $=.5 * \mathrm{X} 1+.5 * \mathrm{X} 2+.8 * \mathrm{X} 3+\mathrm{X} 4+\mathrm{X} 5+.6 * \mathrm{X} 6$

700 LET Z5 $=2.5 * \mathrm{X} 1+3 * \mathrm{X} 2+4 * \mathrm{X} 3+3.5 * \mathrm{X} 4+3 * \mathrm{X} 5+3.6 * \mathrm{X} 6$

710 LET Z6 $=.035 * \mathrm{X} 1+.03 * \mathrm{X} 2+.025 * \mathrm{X} 3+.02 * \mathrm{X} 4+.015 * \mathrm{X} 5+.04 * \mathrm{X} 6$

720 PRINT TAB(1); COUNT; USING "\#\#\#\#\#"; X1; X2; X3; X4; X5; X6; yout; Z1; Z2; Z3; Z4; Z5; Z6

730 RETURN

Programme C.4: A COMPUTER PROGRAMME RESULTS FOR COMPUTING HSLC MIX PROPORTIONS CORRESPONDING TO A REQUIRED CRUSHING STRENGTH

$\begin{array}{llllllllllllll}\text { COUNT } & \mathrm{X} 1 & \mathrm{X} 2 & \mathrm{X} 3 & \mathrm{X} 4 & \mathrm{X} 5 & \mathrm{X} 6 & \mathrm{Y} & \mathrm{Z} 1 & \mathrm{Z} 2 & \mathrm{Z3} & \mathrm{Z} 4 & \mathrm{Z} 5 & \mathrm{Z} 6\end{array}$

$\begin{array}{lllllllllllllll}1 & 0.32 & 0.66 & 0.00 & 0.00 & 0.00 & 0.02 & 49.50 & 0.37 & 1.00 & 0.84 & 0.50 & 2.85 & 0.03\end{array}$

$\begin{array}{lllllllllllllll}2 & & 0.37 & 0.60 & 0.03 & 0.00 & 0.00 & 0.00 & 49.50 & 0.37 & 1.00 & 0.82 & 0.51 & 2.85 & 0.03\end{array}$

$\begin{array}{llllllllllllll}3 & 0.43 & 0.53 & 0.02 & 0.00 & 0.02 & 0.00 & 49.50 & 0.37 & 1.00 & 0.78 & 0.52 & 2.81 & 0.03\end{array}$

$\begin{array}{lllllllllllllll}4 & 0.43 & 0.53 & 0.02 & 0.01 & 0.01 & 0.00 & 49.50 & 0.37 & 1.00 & 0.78 & 0.52 & 2.81 & 0.03\end{array}$

$\begin{array}{lllllllllllllll}5 & & 0.44 & 0.52 & 0.01 & 0.01 & 0.02 & 0.00 & 49.50 & 0.37 & 1.00 & 0.77 & 0.52 & 2.80 & 0.03\end{array}$

$\begin{array}{lllllllllllllll}6 & & 0.44 & 0.52 & 0.01 & 0.02 & 0.01 & 0.00 & 49.50 & 0.37 & 1.00 & 0.77 & 0.52 & 2.80 & 0.03\end{array}$

$\begin{array}{lllllllllllllll}7 & 0.46 & 0.49 & 0.04 & 0.00 & 0.00 & 0.01 & 49.50 & 0.37 & 1.00 & 0.78 & 0.51 & 2.82 & 0.03\end{array}$

$\begin{array}{lllllllllllllll}8 & 0.49 & 0.46 & 0.01 & 0.02 & 0.00 & 0.02 & 49.50 & 0.37 & 1.00 & 0.75 & 0.52 & 2.79 & 0.03\end{array}$

$\begin{array}{lllllllllllllll}9 & & 0.51 & 0.44 & 0.00 & 0.00 & 0.04 & 0.01 & 49.50 & 0.37 & 1.00 & 0.73 & 0.52 & 2.75 & 0.03\end{array}$

$\begin{array}{lllllllllllllll}10 & & 0.51 & 0.44 & 0.03 & 0.00 & 0.02 & 0.00 & 49.50 & 0.37 & 1.00 & 0.74 & 0.52 & 2.78 & 0.03\end{array}$

$\begin{array}{llllllllllllll}11 & 0.52 & 0.42 & 0.02 & 0.00 & 0.00 & 0.04 & 49.50 & 0.37 & 1.00 & 0.75 & 0.51 & 2.78 & 0.03\end{array}$

$\begin{array}{lllllllllllllll}12 & & 0.53 & 0.41 & 0.02 & 0.00 & 0.00 & 0.04 & 49.50 & 0.37 & 1.00 & 0.75 & 0.51 & 2.78 & 0.03\end{array}$

$\begin{array}{lllllllllllllll}13 & 0.54 & 0.41 & 0.02 & 0.00 & 0.03 & 0.00 & 49.50 & 0.37 & 1.00 & 0.72 & 0.52 & 2.75 & 0.03\end{array}$

$\begin{array}{lllllllllllllll}14 & 0.55 & 0.40 & 0.02 & 0.00 & 0.03 & 0.00 & 49.50 & 0.37 & 1.00 & 0.71 & 0.52 & 2.75 & 0.03\end{array}$

$\begin{array}{llllllllllllll}15 & 0.55 & 0.40 & 0.02 & 0.01 & 0.01 & 0.01 & 49.50 & 0.37 & 1.00 & 0.72 & 0.52 & 2.76 & 0.03\end{array}$

$\begin{array}{lllllllllllllll}16 & & 0.55 & 0.41 & 0.00 & 0.04 & 0.00 & 0.00 & 49.50 & 0.37 & 1.00 & 0.71 & 0.52 & 2.75 & 0.03\end{array}$

$\begin{array}{llllllllllllll}17 & 0.57 & 0.38 & 0.03 & 0.00 & 0.02 & 0.00 & 49.50 & 0.37 & 1.00 & 0.71 & 0.52 & 2.75 & 0.03\end{array}$

$\begin{array}{lllllllllllllll}18 & & 0.58 & 0.37 & 0.04 & 0.00 & 0.01 & 0.00 & 49.50 & 0.37 & 1.00 & 0.71 & 0.52 & 2.75 & 0.03\end{array}$

$\begin{array}{lllllllllllllll}19 & & 0.59 & 0.36 & 0.01 & 0.01 & 0.00 & 0.03 & 49.50 & 0.37 & 1.00 & 0.71 & 0.51 & 2.74 & 0.03\end{array}$

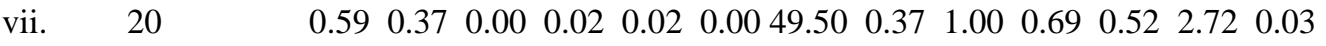

$\begin{array}{llllllllllllllll}21 & 0.60 & 0.36 & 0.02 & 0.02 & 0.00 & 0.00 & 49.50 & 0.37 & 1.00 & 0.69 & 0.52 & 2.73 & 0.03\end{array}$

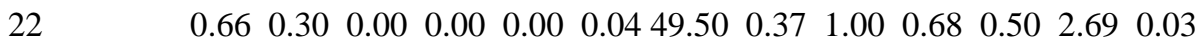

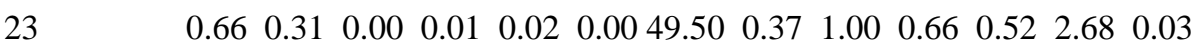

$\begin{array}{lllllllllllllll}24 & & 0.66 & 0.31 & 0.01 & 0.01 & 0.01 & 0.00 & 49.50 & 0.37 & 1.00 & 0.66 & 0.51 & 2.69 & 0.03\end{array}$

$\begin{array}{lllllllllllllll}25 & & 0.66 & 0.31 & 0.02 & 0.01 & 0.00 & 0.00 & 49.50 & 0.37 & 1.00 & 0.67 & 0.51 & 2.70 & 0.03\end{array}$

$\begin{array}{lllllllllllllll}26 & 0.68 & 0.29 & 0.02 & 0.00 & 0.00 & 0.01 & 49.50 & 0.37 & 1.00 & 0.67 & 0.51 & 2.69 & 0.03\end{array}$

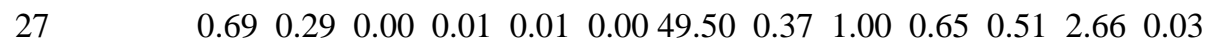

THE MAXIMUM CRUSHING STRENGTH PREDICTABLE BY THIS MODEL

IS 50.05102 N/SQ.MM. 


\section{CONCLUSIONS}

The experimental data are properly fitted to the developed model equation which when tested satisfied the student $-\mathrm{t}$ test. Hence the model equation developed is acceptable for use. For a project requiring high strength of laterised concrete up to and below $50 \mathrm{~N} / \mathrm{mm}^{2}$, the mix proportions to produce such targeted strength can easily be obtained by running the computer programme. The problem of having to go through a rigorous mix-design procedure for a desired strength has been reduced by utilizing the models equation.

\section{REFERENCES}

[1] Akhnazarova, S and Kafarov, V. 1982. Mixture design: simplex lattice method. Experiment Optimization in Chemistry and Chemical Engineering. Trans. Vladimir Matskovsky \& Alexander Repyev. Moscow: MIR Publishers. Rpt.1982. Chapter 6: 240-293.

[2] British Standards Institution, BS812. 1975. Methods of Testing Aggregates. London.

[3] British Standards Institution, BS3148. 1980. Test for Water for Making Concrete (including Notes on the Suitability of the Water), London.

[4] British Standards Institution. BS1881: Part 108. 1983. Method for Making Test Cubes from Fresh Concrete. London.

[5] British Standards Institution. BS1881: Part 116. 1983. Method for Determination of Compressive Strength of Concrete Cubes. London.

[6] British Standards Institution. BS12 1991: Specifications for portland cements. London.

[7] British Standards Institution. BS882: 1992. Specifications for Aggregates from Natural Sources for Concrete. London.

[8] Ettu, L. O; Ibearugbulem, O. M; Ezeh, J. C; \& Anya, U. C. 2013. The Suitability of Using Laterite as Sole Fine Aggregate in Structural Concrete. International Journal of Scientific \& Engineering Research, Volume 4, Issue 5, pp. $45-51$.

[9] Gidigasu, M.D. 1976. Laterite Soil Engineering. Oxford: Elservier Scientific publishers,

[10] Jackson, N. 1983. Civil Engineering Materials. Hong Kong: RDC Arteser Limited.

[11] Orangun, C.O. 1981. Local materials in structural engineering. Proceedings of the symposium on local materials in Civil engineering construction by the Nigerian Society of Engineers (Civil Engineering Division): 20 - 27.

[12] Scheffe, H. 1958. Experiments with mixtures. Royal Statistical Society Journal, B.20: 344-360.

[13] Udoeyo, F; Odim, O. \& Iron, U. 2006. Strength performance of laterized concrete. Construction and Building Materials Journal. 20.10: 1057-1062.

\section{AUTHORS}

First Author - Dr. Udeme Hanson Iron, Lecturer, Department of Civil Engineering, Faculty of Engineering, University of Uyo, Nigeria; 07064658565; hansonudeme@yahoo.com

Second Author - Professor Dahunsi, Bamidele I.O., Professor, Department of Civil Engineering, Faculty of Technology, University of Ibadan, Nigeria; 08033261699; deledahunsi@gmail.com 